\title{
Resonance saturation for four-nucleon operators
}

\author{
Evgeny Epelbaum, ${ }^{1, *}$ Ulf-G. Meißner, ${ }^{1, \dagger}$ Walter Glöckle, ${ }^{2, *}$ and Charlotte Elster ${ }^{1,3, \S}$ \\ ${ }^{1}$ Forschungszentrum Jülich, Institut für Kernphysik (Th), D-52425 Jülich, Germany \\ ${ }^{2}$ Ruhr-Universität Bochum, Institut für Theoretische Physik II, D-44870 Bochum, Germany \\ ${ }^{3}$ Instiute for Nuclear and Particle Physics, Ohio University, Athens, Ohio 45701
}

(Received 6 June 2001; published 6 March 2002)

\begin{abstract}
In the modern description of nuclear forces based on chiral effective field theory, four-nucleon operators with unknown coupling constants appear. These couplings can be fixed by a fit to the low partial waves of nucleon-nucleon scattering. We show that the so-determined numerical values have a remarkable similarity to values extracted from phenomenological one-boson-exchange models in a low momentum expansion. We also extract these values from various modern high accuracy nucleon-nucleon potentials and find again the same similarity. This paves the way for estimating the low-energy constants of operators with more nucleon fields and/or external probes.
\end{abstract}

DOI: 10.1103/PhysRevC.65.044001

PACS number(s): 13.20.Gd, 12.39.Fe, 24.80.+y, 24.85.+p

\section{INTRODUCTION}

Effective chiral Lagrangians can be used to investigate the dynamics of pion and pion-nucleon as well as nucleonnucleon interactions. In all cases, one has to consider two distinct contributions, namely tree and loop diagrams, which are organized according to the underlying power counting $[1,2]$. To a given order, one has to consider all local operators constructed from pions, nucleon fields, and external sources in harmony with chiral symmetry, Lorentz invariance, and the pertinent discrete symmetries. Beyond (or even at) leading order in the chiral expansion, these operators are accompanied by unknown coupling constants, also called lowenergy constants (LECs). In principle, these LECs are calculable from QCD but in practice need to be fixed by a fit to some data or using some model. ${ }^{1}$ While in certain cases sufficient data exist allowing one to pin down the LECs, often some good estimate for these constants beyond naive dimensional analysis is needed. In the meson sector, the ten LECs of the chiral Lagrangian at next-to-leading order (NLO) have been determined [2], and their values can be understood in terms of masses and coupling constants of the lowest meson resonances of vector, axial-vector, scalar, and pseudoscalar character, maybe with the exception of the scalar sector with vacuum quantum numbers $[5,6]$. This is called resonance saturation, it has been used, e.g., to estimate LECs at next-to-next-to-leading order (NNLO) (see, e.g., Ref. [7]) or for the extended chiral Lagrangian including virtual photons as dynamical degrees of freedom $[8] .^{2} \mathrm{~A}$ similar system-

\footnotetext{
*Email address: evgeni.epelbaum@tp2.ruhr-uni-bochum.de

${ }^{\dagger}$ Email address: u.meissner@fz-juelich.de

\#Email address: walter.gloeckle@tp2.ruhr-uni-bochum.de

${ }^{\S}$ Email address: c.elster@fz-juelich.de

${ }^{1}$ These LECs can also be calculated in lattice gauge theory. For a first attempt in the Goldstone boson sector using the strong coupling expansion see Ref. [3], while the most recent quenched calculation for these LECs is given in Ref. [4].

${ }^{2}$ For a critical discussion of resonance saturation concerning these LECs, see Ref. [9]. Note also that the status of resonance saturation
}

atic analysis exists for the finite dimension-two couplings of the pion-nucleon effective Lagrangian [11], where it was demonstrated the LECs are saturated in terms of baryon resonance excitation in the $s-, u$-, and $t$-channel meson resonances. Much less is known about dimension three and four couplings, but for certain processes resonance saturation has been shown to work quite well, e.g., in neutral pion photoproduction off protons [12]. A somewhat different scheme (including also meson-resonance loops) was introduced in the study of the baryon octet masses in Ref. [13]. The situation is very different concerning few-nucleon systems, where a new type of operators with $2 A$ nucleon fields appears (for reactions involving $A \geqslant 2$ nucleons). Only recently, a complete and precise determination of the four $S$-wave and five $P$-wave LECs in neutron-proton scattering has become available [14]; thus it is timely to ask the question whether the numerical values of these four-nucleon coupling constants can be at least qualitatively understood from some kind of resonance saturation. ${ }^{3}$ This will be the topic of the present paper.

The traditional approach to nuclear forces is the exchange of pions and heavier mesons and the consideration of excited states of the nucleon. The simplest, rather successful approach, considering only exchanges of single mesons and neglecting all excited states of the nucleon, is the one-bosonexchange model (OBE). If fine tuned it provides in the form of the CD Bonn [18] or Nijmegen I, II [19] potentials an essentially perfect description of the rich set of nucleonnucleon $(N N)$ data. We pose the question whether the physical ingredients of these models (meson masses, coupling constants, strong form factor parameters) can be related to the LECs of the chiral $N N$ forces. To that aim we perform a low-momentum expansion of the various heavy-meson ex-

for the nonleptonic weak LECs is less clear [10].

${ }^{3}$ Note that in the pioneering work [16] global fits with 26 free parameters where performed, which presumably do not allow to pin down the LECs in a unique way. For more details and further discussion on various differences between our formalism and the one of Ref. [16], see Refs. [17,14]. 
changes. This leads to a string of contact forces with increasing powers of momenta added to the one-pion exchange. That form can be compared to the $N N$ forces determined in chiral perturbation theory, which parametrizes short range physics in terms of a corresponding string of contact forces accompanied by the LECs and explicit one- and more-pion exchanges. The latter can be equally expanded in order to enable a direct comparison of contact forces in both approaches. In this first study we do not address the question, whether such a comparison is not somewhat blurred by the fact that the typical momentum range of the OBE potentials and the chiral $N N$ forces are different. The momentum range smoothly cut off for OBE potentials is typically $1-2 \mathrm{GeV}$, whereas for the chiral $N N$ forces considered here it is 500-600 MeV. A presumably more adequate comparison would be to rewrite the OBE force into phase equivalent effective ones belonging to the same low-momentum range as the chiral one. This can be achieved along the line worked out in Ref. [20]. Since we do not expect a qualitative change of the results in which we are only interested in this first study, we refrain from doing that.

For the comparison it is important that the chiral forces do not support deeply bound, spurious states, which can occur in the effective field theory (EFT) approach [14] and are not relevant, since they lie outside the theory's domain of validity. Their existence, however, destroys the phase equivalence to the OBE models, which are free of these spurious states. To get rid of spurious bound states in the NNLO chiral potential we had to make a specific choice for the subleading LECs $c_{3}$ and $c_{4}$ which occur at NNLO and drive the $\pi \pi$ coupling to the nucleon. In principle, they should be fixed in the $\pi N$ system. As we found in Ref. [14], taking the numerically quite large values of the $c_{i}$ 's from the $Q^{3}$ analysis of the $\pi N$ system does not allow us to fit the $N N$ data without introducing the unphysical deeply bound states. The dominant part of the $c_{3}$ and $c_{4}$ is known to be due to an intermediate $\Delta[11]$, which in the $N N$ system correspond to diagrams with intermediate $\Delta$ states. In the meson exchange picture of the $N N$ force it is well established that graphs with intermediate $\Delta$-isobar states and pion exchange are overly attractive in low partial waves. This attraction is counterbalanced by an additional $\pi \rho$ exchange with intermediate $\Delta$ states $[21,22]$. In order to account for this cancellation in the EFT we make the choice to subtract out the $\Delta$ contribution in the $c_{i}$ values. This leads to a novel description to calculate the NNLO chiral effective field theory potential, which is free of deeply bound spurious $N N$ states and leads to an equally good description of the $N N$ phase shifts as using the unsubtracted $c_{i}$ values $[14,15]$. Clearly this choice of the $c_{i}$ values requires more investigations in the future by understanding better the role of the intermediate $\Delta$ excitation at low energies, where the kinematics is apparently quite different in the $N N$ system compared to the $\pi N$ system. With all that in mind we can obtain the LECs from the OBE models in terms of their resonance parameters, and find them strikingly similar to the ones determined in the EFT picture directly from the $N N$ data. In a further step we extended our investigation to other modern $N N$ force models of more phenomenological character, the Argonne V18 [38] and the
Nijmegen I and II interactions [19], with equally perfect descriptions of $N N$ scattering data. This group, including CDBonn, Nijmegen I,II, Argonne V18 potentials is often referred to as high-precision $N N$ potentials. We find similar results as for the OBE potentials. This paves the way for estimating the low-energy constants of operators with more nucleon fields and/or external probes. We mention that in studies of pion production in proton-proton collisions or charge symmetry breaking in the $N N$ interaction, ideas of resonance saturation have already been used $[23,24]$. Also, Friar [25] has discussed aspects of integrating out heavymeson fields to generate local four-nucleon operators with given LECs but did not attempt a detailed comparison with existing models of the nuclear forces as done here.

The outline of the article is as follows. In Sec. II we discuss the effective chiral Lagrangian for nucleon-nucleon interactions, in particular the four-nucleon terms and their corresponding coupling constants. We then summarize how these LECs are determined at NLO and give a novel prescription to calcuate the NNLO chiral effective field theory (EFT) potential. In Sec. III we show how to calculate these LECs from existing boson-exchange or phenomenological potentials and compare the resulting values with the ones obtained in EFT. Section IV is devoted to the study of the naturalness of these coupling constants and the implications of Wigner's spin-isospin symmetry. Our conclusions are summarized in Sec. V. Some technicalities are relegated to Appendices.

\section{CHIRAL EFFECTIVE FIELD THEORY}

\section{A. Effective Lagrangian and definition of LECs}

To be specific, we briefly discuss the approach to chiral Lagrangians for few-nucleon systems proposed by Weinberg. One starts from an effective chiral Lagrangian of pions and nucleons, including in particular local four-nucleon interactions that describe the short-range part of the nuclear force, symbolically

$$
\mathcal{L}_{\text {eff }}=\mathcal{L}_{\pi \pi}+\mathcal{L}_{\pi N}+\mathcal{L}_{N N}
$$

where each of the terms admits an expansion in small momenta and quark (meson) masses. To a given order, one has to include all terms consistent with chiral symmetry, parity, charge conjugation, and so on. The last term in Eq. (1) contains the four-, six-, . . . nucleon terms of interest here. From the effective Lagrangian, one derives the two-nucleon potential. This potential is based on (a modified) Weinberg counting [17]; more precisely, one organizes the unitarily transformed infrared nonsingular diagrams according to their power (chiral dimension) in small momenta and pion masses (for a detailed discussion, see Ref. [17]). To leading order (LO), this potential is the sum of one-pion exchange (OPE) (with pointlike coupling) and of two four-nucleon contact interactions without derivatives. The low-energy constants accompanying these terms have to be determined by a fit to some data, such as the two $S$-wave phase shifts in the lowenergy region (for $n p$ ). At next-to-leading order (NLO), one has corrections to the OPE, the leading order two-pion ex- 
change graphs and seven-dimensional two four-nucleon terms with unknown LECs (for the $n p$ system). Finally, at NNLO, one has further renormalizations of the one- and corrections to the two-pion exchange graphs including dimension-two pion-nucleon operators. The corresponding LECs can be determined from the chiral perturbation theory (CHPT) analysis of pion-nucleon scattering. The existence of shallow nuclear bound states (and large scattering lengths) forces one to perform an additional nonperturbative resummation. This is done here by obtaining the bound and scattering states from the solution of the Lippmann-Schwinger equation. The potential has to be understood as regularized, and the regularization is dictated by the EFT approach employed here, i.e.,

$$
V\left(p, p^{\prime}\right) \rightarrow f_{R}(p) V\left(p, p^{\prime}\right) f_{R}\left(p^{\prime}\right),
$$

where $f_{R}(p)$ is a regulator function chosen in harmony with the underlying symmetries. Within a certain range of cutoff values, the physics should be independent of its precise form and value [26]. That this is indeed the case has been demonstrated in Ref. [14]. The central object of the study presented here are the LECs related to the four-nucleon operators. In a spectroscopic notation these are called $C_{1 S_{0}}, \widetilde{C}_{{ }_{1} S_{0}} C_{{ }^{3} S_{1}}$, $\widetilde{C}_{{ }^{3} S_{1}}, C_{3 D_{1}-{ }^{3} S_{1}}:=C_{\epsilon_{1}}, C_{{ }_{1} P_{1}}, C^{3} P_{0}, C^{3} P_{1}$, and $C_{3 P_{2}}$. In the following, we will collectively denote these as $C_{i}$ and $\widetilde{C}_{i}$, respectively. The two LECs $\widetilde{C}_{i}$ stem from the two momentum-independent four-nucleon operators, while the seven $C_{i}$ are related to two-derivative operators as they appear in the effective Lagrangian (we have adopted the notation to the two-nucleon potential given in Ref. [27]),

$$
\begin{gathered}
\mathcal{L}_{N N}=\mathcal{L}_{N N}^{(2)}+\mathcal{L}_{N N}^{(4)}+\cdots, \\
\mathcal{L}_{N N}^{(2)}=-\frac{1}{2} C_{S}\left(N^{\dagger} N\right)\left(N^{\dagger} N\right)-\frac{1}{2} C_{T}\left(N^{\dagger} \sigma_{i} N\right)\left(N^{\dagger} \sigma_{i} N\right), \\
\mathcal{L}_{N N}^{(4)}=-\frac{1}{2} C_{1}\left\{\left(N^{\dagger} \partial_{i} N\right)^{2}+\left[\left(\partial_{i} N^{\dagger}\right) N\right]^{2}\right\}-\left(C_{1}-\frac{1}{4} C_{2}\right)\left(N^{\dagger} \partial_{i} N\right)\left[\left(\partial_{i} N^{\dagger}\right) N\right]+\frac{1}{8} C_{2}\left(N^{\dagger} N\right)\left[N^{\dagger} \partial_{i}^{2} N+\partial_{i}^{2} N^{\dagger} N\right] \\
-\frac{i}{8} C_{5} \epsilon_{i j k}\left(\left\{\left(N^{\dagger} \partial_{i} N\right)\left[\left(\partial_{j} N^{\dagger}\right) \sigma_{k} N\right]+\left[\left(\partial_{i} N^{\dagger}\right) N\right]\left(N^{\dagger} \sigma_{j} \partial_{k} N\right)\right\}-\left(N^{\dagger} N\right)\left[\left(\partial_{i} N^{\dagger}\right) \sigma_{j} \partial_{k} N\right]+\left(N^{\dagger} \sigma_{i} N\right)\left[\left(\partial_{j} N^{\dagger}\right) \partial_{k} N\right]\right) \\
+\frac{1}{4}\left[\left(C_{6}+\frac{1}{4} C_{7}\right)\left(\delta_{i k} \delta_{j l}+\delta_{i l} \delta_{k j}\right)+\left(2 C_{3}+\frac{1}{2} C_{4}\right) \delta_{i j} \delta_{k l}\right]\left\{\left[\left(\partial_{i} \partial_{j} N^{\dagger}\right) \sigma_{k} N\right]+\left(N^{\dagger} \sigma_{k} \partial_{i} \partial_{j} N\right)\right\}\left(N^{\dagger} \sigma_{l} N\right) \\
-\frac{1}{2}\left[C_{6}\left(\delta_{i k} \delta_{j l}+\delta_{i l} \delta_{k j}\right)+C_{4} \delta_{i j} \delta_{k l}\right]\left(N^{\dagger} \sigma_{k} \partial_{i} N\right)\left[\left(\partial_{j} N^{\dagger}\right) \sigma_{l} N\right]-\frac{1}{8}\left(\frac{1}{2} C_{7}\left(\delta_{i k} \delta_{j l}+\delta_{i l} \delta_{k j}\right)-\left(4 C_{3}-3 C_{4}\right) \delta_{i j} \delta_{k l}\right) \\
\times\left[\left(\partial_{i} N^{\dagger} \sigma_{k} \partial_{j} N\right)+\left(\partial_{j} N^{\dagger} \sigma_{k} \partial_{i} N\right)\right]\left(N^{\dagger} \sigma_{l} N\right),
\end{gathered}
$$

where $N$ denotes the (nonrelativistic) nucleon fields, $N=(p, n)^{T}, \sigma_{l}(l=1,2,3)$ are the Pauli spin matrices, and the summation convention for repeated indices is understood. Since we are not considering external sources here, we only have partial derivatives acting on the nucleon fields. To arrive at this expression for the most general effective Lagrangian with four nucleon field operators, we have made use of partial integration, Fierz transformation, and the equation of motion for the nucleons. We also require reparametrization invariance [28] of the Lagrangian, which allows us to further reduce the number of independent terms as compared to Ref. [16]. The complete derivation of Eq. (3) within the heavy-baryon formalism is presented in Ref. [27]. Note that the effective Lagrangian (3) corresponds to the rest-frame system of the nucleon with the velocity operator given by $v_{\mu}=(1,0,0,0)$. The resulting $N N$ contact potential reads [in the center-of-mass (c.m.) system]

$$
\begin{aligned}
V_{\text {cont }}= & C_{S}+C_{T}\left(\vec{\sigma}_{1} \cdot \vec{\sigma}_{2}\right)+C_{1} \vec{q}^{2}+C_{2} \vec{k}^{2}+\left(C_{3} \vec{q}^{2}+C_{4} \vec{k}^{2}\right)\left(\vec{\sigma}_{1} \cdot \vec{\sigma}_{2}\right)+i C_{5} \frac{\vec{\sigma}_{1}+\vec{\sigma}_{2}}{2} \cdot(\vec{q} \times \vec{k})+C_{6}\left(\vec{q} \cdot \overrightarrow{\sigma_{1}}\right)\left(\vec{q} \cdot \vec{\sigma}_{2}\right)+C_{7}\left(\vec{k} \cdot \overrightarrow{\sigma_{1}}\right) \\
& \times\left(\vec{k} \cdot \vec{\sigma}_{2}\right)
\end{aligned}
$$

where $\vec{q}=\vec{p}^{\prime}-\vec{p}$ and $\vec{k}=\left(\vec{p}+\vec{p}^{\prime}\right) / 2$ are the transferred and the averaged momentum, respectively, and $\vec{p}\left(\vec{p}^{\prime}\right)$ corresponds to the initial (final) momentum of the nucleons in the c.m. system. Closer inspection of Eq. (4) might lead to the question why no operators containing the isospin matrices $\tau_{i}$ (where $i=1,2$ labels the nucleons) appear For example, $\rho$-meson exchange will naturally lead to a contribution $\sim \boldsymbol{\tau}_{1} \cdot \boldsymbol{\tau}_{2}$. In principle, at NLO, one can write down 18 operators in the effective potential and not just nine as appear here. What seems to be completely missing are the nine operators 
TABLE I. Values of the LECs at NLO and NNLO for the cutoff values $\Lambda=500, \ldots, 600 \mathrm{MeV}$. Also given are the contributions from two-pion exchange at NLO and NNLO that are contained in the values of the LECs as explained in the text. The $\widetilde{C}_{i}$ are in $10^{4} \mathrm{GeV}^{-2}$ and the $C_{i}$ in $10^{4} \mathrm{GeV}^{-4}$.

\begin{tabular}{|c|c|c|c|c|}
\hline LEC & TPE(NLO) & TPE(NNLO) & $C_{i}(\mathrm{NLO})$ & $C_{i}(\mathrm{NNLO})$ \\
\hline$\widetilde{C}_{{ }_{1} S_{0}}$ & -0.004 & 0.003 & $-0.156, \ldots,-0.110$ & $-0.160, \ldots,-0.158$ \\
\hline$C_{1}$ & -0.585 & -0.070 & $1.048, \ldots, 1.253$ & $1.135, \ldots, 1.134$ \\
\hline$\widetilde{C}_{3 S_{1}}$ & 0.013 & 0.001 & $-0.155, \ldots,-0.023$ & $-0.159, \ldots,-0.134$ \\
\hline$C_{3 S_{1}}$ & 0.653 & -0.181 & $0.250, \ldots, 0.840$ & $0.637, \ldots, 0.587$ \\
\hline$C_{\epsilon_{1}}$ & -0.195 & 0.117 & $-0.302, \ldots,-0.384$ & $-0.369, \ldots,-0.326$ \\
\hline$C_{1} P_{1}$ & -0.069 & -0.099 & $0.260, \ldots, 0.273$ & $0.234, \ldots, 0.268$ \\
\hline$C_{3} P_{0}$ & -0.436 & -0.071 & $0.800, \ldots, 0.855$ & $0.727, \ldots, 0.857$ \\
\hline$C_{3} P_{1}$ & 0.252 & 0.011 & $-0.126, \ldots,-0.093$ & $-0.141, \ldots, 0.026$ \\
\hline $\mathrm{C}_{3} \mathrm{P}_{2}$ & -0.023 & 0.036 & $-0.325, \ldots,-0.259$ & $-0.464, \ldots,-0.445$ \\
\hline
\end{tabular}

involving products of isospin matrices. However, we remind the reader that only nine of these 18 operators are independent. The terms in the Lagrangian related to the other nine can be eliminated using Fierz transformations $[1,17]$. Equivalently, one can perform an antisymmetrization of the two-nucleon potential to eliminate redundant terms as used in Refs. [29,14,27]. Clearly, the set of operators we choose to work with is one but not the unique possibility.

As stated before, there are two (seven) LECs related to operators with zero (two) derivatives. These constants can be most easily determined by a fit to the $S$ - and $P$-wave phase shifts and the ${ }^{3} S_{1}-{ }^{3} D_{1}$ mixing parameter at low energies, which leads naturally to certain linear combinations, i.e., the already enumerated spectroscopic LECs. The precise relation of the LECs appearing in the effective Lagrangian to the spectroscopic ones is taken from Ref. [14] (correcting some typographical errors in that reference),

$$
\begin{aligned}
& \widetilde{C}_{{ }_{1} S_{0}}=4 \pi\left(C_{S}-3 C_{T}\right), \\
& C^{{ }_{1} S_{0}}=\pi\left(4 C_{1}+C_{2}-12 C_{3}-3 C_{4}-4 C_{6}-C_{7}\right), \\
& \widetilde{C}_{{ }_{3}}=4 \pi\left(C_{S}+C_{T}\right), \\
& C_{3} S_{1}=\frac{\pi}{3}\left(12 C_{1}+3 C_{2}+12 C_{3}+3 C_{4}+4 C_{6}+C_{7}\right), \\
& C_{1} P_{1}=\frac{2 \pi}{3}\left(-4 C_{1}+C_{2}+12 C_{3}-3 C_{4}+4 C_{6}-C_{7}\right), \\
& C_{3 P_{1}}=\frac{2 \pi}{3}\left(-4 C_{1}+C_{2}-4 C_{3}+C_{4}+2 C_{5}-8 C_{6}+2 C_{7}\right), \\
& C_{3 P_{2}}=\frac{2 \pi}{3}\left(-4 C_{1}+C_{2}-4 C_{3}+C_{4}-2 C_{5}\right), \\
& C_{3} P_{0}=\frac{2 \pi}{3}\left(-4 C_{1}+C_{2}-4 C_{3}+C_{4}+4 C_{5}+12 C_{6}-3 C_{7}\right),
\end{aligned}
$$

$$
C^{3 D_{1}{ }^{-3} S_{1}}=C_{\epsilon_{1}}=\frac{2 \sqrt{2} \pi}{3}\left(4 C_{6}+C_{7}\right) .
$$

\section{B. LECs at next-to-leading order}

Let us now discuss the determination of NLO LECs of the chiral EFT potential. In contrast to what was done in Ref. [14], we also include the leading charge dependence effect, which is the charged to neutral pion mass difference, $\Delta M_{\pi}$ $=M_{\pi^{ \pm}}-M_{\pi^{0}}$, in the OPE potential (for a systematic study of such effects, see Ref. [30].) Fitting the low neutron-proton $(n p)$ partial waves $(S, P$ and the triplet $S-D$ mixing) for center-of-mass energies below 50-100 MeV, one obtains the numerical values of the LECs for the given regulator and cutoff value. We work here with an exponential regulator,

$$
f_{R}(p)=\exp \left(-p^{4} / \Lambda^{4}\right)
$$

where the momentum cutoff $\Lambda$ is varied between 500 and $600 \mathrm{MeV}$ (a more detailed discussion of various regulator functions is given in Ref. [14]). Therefore, we obtain a range of values for each LEC in the given partial waves. For a direct comparison with one-boson-exchange models, we need to further add the two-pion exchange (TPE) contribution, which stems from the box, triangle, and football diagrams. This is done by expanding the contributions of these graphs in terms of local operators with increasing powers of derivatives and projecting onto the appropriate partial waves (this method is described in more detail below). The so obtained numerical contributions in each partial wave are listed in Table I in the column TPE (NLO) (for explicit analytical expressions, see Appendix A). Obviously, these numbers are cutoff independent.

In contrast, the OPE is retained because all potentials we will compare to include it as well. We note that some of these potentials contain a pion-nucleon form factor, but since it only depends on the momentum transfer squared and appears quadratically, it does not influence any four-nucleon operator with zero or two derivatives. With this in mind, we present in Table I the resulting values of the LECs for the cutoff varying from 500 to $600 \mathrm{MeV}$. This is the optimal range found in the study of few-nucleon system $[14,31]$ as well as proton- 
proton scattering [30] in the framework used here. For more discussion about the choice of the cutoff in the EFT see Ref. [26]. Note that in principle we could take smaller values for the cutoff. In such a case one would get a slightly less precise description of the data. Lowering the $\Lambda$ value too much would, however, result in losing the interesting physics of many-meson exchanges, which is driven by chiral symmetry and which is parameter free. Also it is clear that due to our choice of the regulating function, Eq. (6), one would have to restrict oneself to a smaller energy range when choosing $\Lambda$ $<500 \mathrm{MeV}$. For example, at the energy $E_{\mathrm{lab}}=300 \mathrm{MeV}$, which corresponds to a c.m. momentum of about $375 \mathrm{MeV}$, one has for $\Lambda=400 \mathrm{MeV} f_{R} \sim 0.7$. Thus, significant deviations from the data would appear at these energies at least in the partial waves with large angular momenta, which are governed by the OPE and TPE and do not require iteration of the potential. Since we want to have a quantitatively good description of the data also at intermediate energies up to $E_{\text {lab }}=200 \mathrm{MeV}$, we refrain from lowering $\Lambda$ beyond $500 \mathrm{MeV}$. The sharp cutoff might be a better choice for studies with smaller values of $\Lambda$. Further, one could not substantially increase the cutoff values if no unphysical deeply bound states are allowed $[14,32] .{ }^{4}$ Also from physical reasons it would probably not make much sense to further increase the values of the cutoff without explicit treatment of the heavy degrees of freedom [26]. Important is the fact that the $\Lambda$ dependence should get weaker and weaker with increasing order in the expansion. We clearly see this effect up to the order we have explored the EFT approach. Last but not least, a similar upper bound for $\Lambda$ was also found in Ref. [33].

\section{Phase-equivalent potentials and LECs at next-to-next-to-leading order}

We now turn to the determination of the LECs based on the NNLO potential. Here, we perform a modification as compared to the work presented in Ref. [14]. At that order, the pion-nucleon LECs $c_{1,3,4}$ appear, which have been taken from the CHPT analysis of $\pi N$ scattering in the interior of the Mandelstam triangle [34]. As already shown in Ref. [11], these values can be understood in terms of baryon and meson resonance excitations, with a particularly strong contribution from the $\Delta(1232)$ resonance. While the natural size for these LECs is $1 \mathrm{GeV}^{-1}$, typical values found for $c_{3}$ and $c_{4}$ from $\pi N$ scattering data are $c_{3}=(-4.70 \pm 1.16) \mathrm{GeV}^{-1}$ and $c_{4}$ $=(3.40 \pm 0.04) \mathrm{GeV}^{-1}$, respectively. The resulting TPE with insertion of these operators improves the fit but leads to a very strongly attractive central potential, as witnessed by the appearance of deeply bound states, e.g., in the deuteron channel. These states do, however, not influence the lowenergy physics in the two-nucleon system. However, the resulting potential is clearly not phase equivalent to the one-

\footnotetext{
${ }^{4}$ Although, as will be stressed in Sec. II C, such spurious bound states would not affect low-energy $N N$ observables, direct comparison with the realistic $N N$ potentials would not be possible.
}
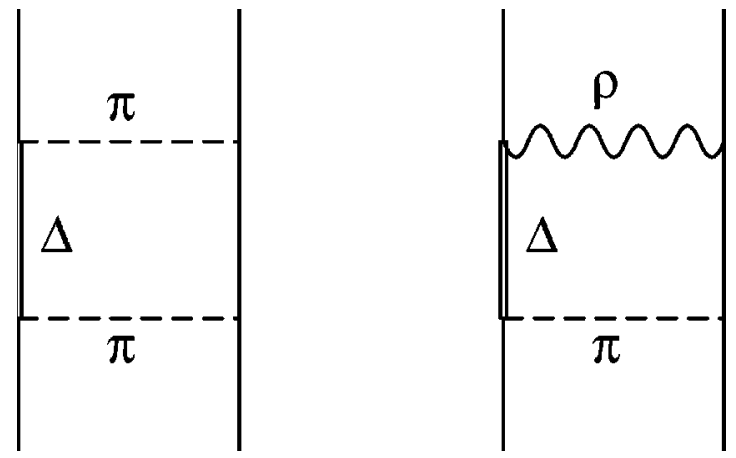

FIG. 1. Classes of diagrams that cancel to large extent. One representative TPE graph and one $\pi \rho$ graph are shown. Solid, double, dashed, and wiggly lines represent nucleons, $\Delta$, pions, and $\rho$ mesons, respectively.

boson-exchange or phenomenological potentials, in which the parameters are tuned in a way that no such additional bound states appear.

There is also a more microscopic argument. In models including the $\Delta(1232)$ resonance explicitly (like the Bonn [22] or Utrecht [21] models), such strongly attractive contributions stem from the TPE with intermediate $\Delta$ states. In low partial waves these graphs are almost completely canceled by graphs with intermediate $\Delta$ states in which one of the pions is replaced by a $\rho$ meson; see Fig. 1. It is even stated in Ref. [22] that "the $2 \pi$ contribution appears, in general, too attractive and a consistent and quantitative description of all phase shifts can never be reached." Further work on a detailed understanding of correlated $\pi \rho$ exchange has been performed by Holinde and co-workers; see Ref. [35]. In the EFT approach, the precise order in which the $\pi \rho$ diagrams with intermediate $\Delta$ start contributing to four-nucleon operators depends on the representation of the vector fields (and thus need not appear at the same order as the corresponding $2 \pi$ graphs). In order to account for the described cancellation and to avoid appearance of deeply bound states in the low partial waves, we have constructed a new chiral potential at NNLO wherein the NNLO TPE graphs we have substituted the $\pi N$ LECs,

$$
c_{i} \rightarrow \tilde{c}_{i}=c_{i}-c_{i}^{\Delta}, \quad i=3,4
$$

using the formalism of Ref. [11] to calculate the $c_{i}^{\Delta}$. More precisely, we have allowed for some fine tuning of the $\tilde{c}_{i}$ within the bounds given in that reference. By this method, the equivalent TPE graphs with intermediate $\Delta$ are subtracted and the aforementioned cancellations are effectively taken into account. For a typical NNLO fit, we use $c_{1}=$ $-0.81 \mathrm{GeV}^{-1}, c_{3}=-1.15 \mathrm{GeV}^{-1}$, and $c_{4}=1.20 \mathrm{GeV}^{-1}$. From that, we obtain the NNLO TPE contribution listed in Table I (for explicit analytical expressions, see Appendix A). A more detailed description of this procedure and further justification of it as well as discussion of the uncertainty in determination of the $c_{i}$ 's are given in Refs. [36,15]. The so determined TPE NNLO contribution and the corresponding LECs are displayed in Table I. It is important to note that in the cases where the TPE contribution is large, the NNLO 

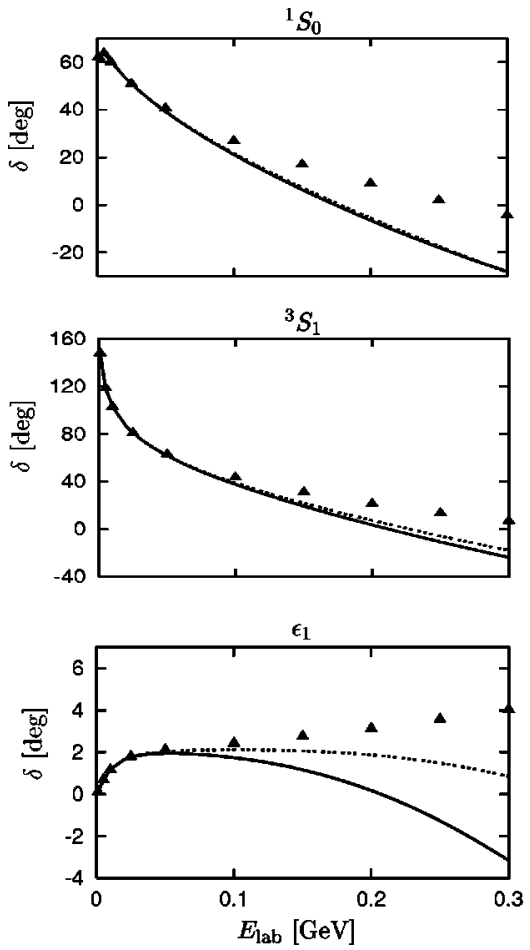
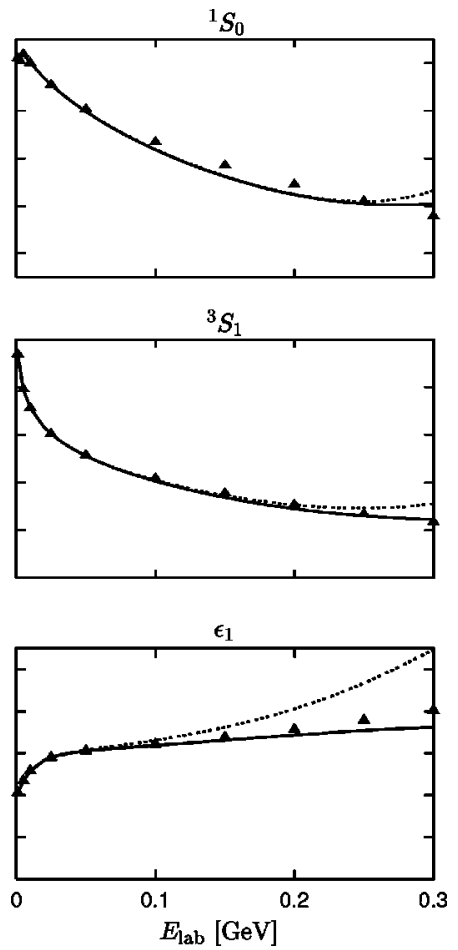

FIG. 2. Phase shifts at NLO (left panel) and NNLO (right panel) versus the laboratory energy (in $\mathrm{GeV}$ ) in comparison to the Nijmegen PSA (filled triangles). The solid (dotted) line corresponds to $\Lambda=500(\Lambda=600) \mathrm{MeV}$. correction is sizably smaller than the NLO one. The resulting values for the LECs $C_{i}$ and $\widetilde{C}_{i}$ at NNLO are consistent with the ones found at NLO. That is an important result.

Before procceding, we would like to stress that the NNLO potential with the reduced values of the LECs $c_{3,4}$ describes the phase shifts with the precision comparable to the NNLO result of Ref. [14]. In Fig. 2 we show the two $n p S$-wave phase shifts ${ }^{1} S_{0}$ and ${ }^{3} S_{1}$ and the ${ }^{3} S_{1^{-}}{ }^{3} D_{1}$ mixing parameter at NLO (left panel) and NNLO (right panel) in comparison to the Nijmegen phase shift analysis (PSA). To regularize the LS equation, we have used an exponential regulator $f_{R}(\vec{p})$ $=\exp \left(-p^{4} / \Lambda^{4}\right)$. The two lines correspond to cutoffs $\Lambda$ $=500$ and $600 \mathrm{MeV}$. We note that the description of the phases improves when going from NLO to NNLO and that also the cutoff dependence gets weaker (especially at low energies). This is to be expected from a converging EFT [26] and we emphasize again that this is not the result of an increasing number of free parameters. A direct comparison with Figs. 4 and 5 of Ref. [14] indeed shows that these phases are reproduced with the precision comparable to the NNLO calculation with the larger values for the $c_{i}$. For the other phase shifts and a more detailed discussion, see Ref. [15].

We are now in the position to confront the LECs determined from chiral effective field theory with the highly successful phenomenological/meson models of the nuclear force. Before doing that, some discussion concerning the NNLO potential constructed in Ref. [14] is in order. It is a perfectly viable scenario to use the unsubtracted values for the $c_{i}$ as done there, since the resulting deep bound states do not influence the physics in the two-nucleon system. As noted already, a direct comparison of the contact terms in the potential with the ones obtained from the meson-exchange or phenomenological approaches cannot be made. While the physics in three- or four-nucleon systems does not depend on the choice of the unsubtracted or subtracted $c_{i}$, the latter choice is closer to standard nuclear physics in which threebody forces lead to small binding energy corrections [15]. In fact, applying directly the potential from Ref. [14] to such systems leads to much smaller binding energies from the two-nucleon forces alone. However, such a separation of the total binding energy into $N N$ and $3 N$ contributions is not observable and therefore this scenario is not ruled out. These topics will be discussed in much more detail in Ref. [15]. At this stage, both options discussed here are viable. It is fair to say that more detailed calculations in few-nucleon systems have to be performed to ultimately clarify this issue. We proceed using the modified dimension-two pion-nucleon couplings; cf. Eq. (7).

\section{LECS FROM BOSON EXCHANGE AND PHENOMENOLOGICAL $N N$ POTENTIALS}

We consider first genuine one-boson-exchange models of the $N N$ force, in which the long-range part of the interaction is given by OPE (including in general a pion-nucleon form factor), whereas shorter distance physics is expressed in terms of a sum over heavier mesons,

$$
V_{N N}=V_{\pi}+\sum_{M=\sigma, \rho, \ldots} V_{M}
$$

where some mesons can be linked to real resonances (such as the $\rho$ meson) or are parametrizations of certain physical effects, e.g., the light scalar-isoscalar $\sigma$ meson is needed to supply the intermediate-range attraction (but it is not a resonance). The corresponding meson-nucleon vertices are given in terms of one (or two) coupling constant(s) and corresponding form factor(s), characterized by some cutoff $\Lambda_{M}$. 


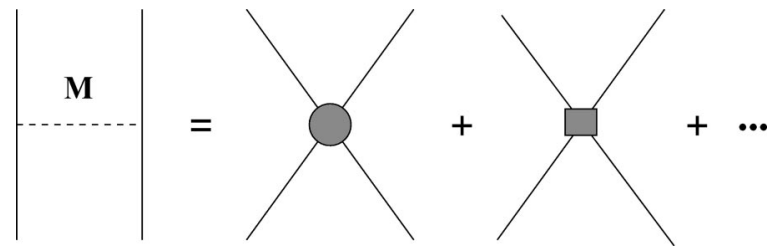

FIG. 3. Expansion of a meson-exchange diagram in terms of local four-nucleon operators. The dashed and solid lines denote the meson $M=\rho, \sigma, \omega, \ldots$ and the nucleons, respectively. The blob and the square denote insertions with zero and two derivatives, in order. The ellipses stands for operators with more derivatives.

These form factors are needed to regularize the potential at small distances (large momenta) but they should not be given a physical interpretation. As depicted in Fig. 3, in the limit of large meson masses, keeping the ratio of coupling constant to mass fixed, one can interpret such exchange diagrams as a sum of local operators with increasing number of derivatives (momentum insertions). In a highly symbolic relativistic notation, this reads

$$
\begin{aligned}
\left(\bar{N} P_{i} N\right)\left(\frac{g^{2} \delta^{i j}}{M_{R}^{2}-t}\right)\left(\bar{N} P_{j} N\right)= & \left(\frac{g^{2}}{M_{R}^{2}}\right)\left(\bar{N} P_{i} N\right)\left(\bar{N} P^{i} N\right)+\left(\frac{g^{2} t}{M_{R}^{4}}\right) \\
& \times\left(\bar{N} P_{i} N\right)\left(\bar{N} P^{i} N\right)+\cdots
\end{aligned}
$$

where the $P_{i}$ are projectors on the appropriate quantum numbers for a given meson exchange (including also Dirac matrices if needed) and $M_{R}$ is the mass of the corresponding heavy meson. It should be kept in mind here that one usually makes use of the nonrelativistic expansion, i.e., the Dirac spinors on the right-hand side of Eq. (9) coincide with the Pauli spinors. In the case of a momentum-dependent mesonnucleon coupling, such as for a monopole form factor normalized to one at $t=0$,

$$
g(t)=g \frac{\Lambda_{M}^{2}}{\Lambda_{M}^{2}-t},
$$

then the coefficient of the first $t$-dependent term in Eq. (9) is modified to

$$
\frac{g^{2}}{M_{R}^{4}} \rightarrow \frac{g^{2}}{M_{R}^{2}}\left(\frac{1}{M_{R}^{2}}+\frac{2}{\Lambda_{M}^{2}}\right),
$$

and accordingly for other types of form factors (dipole, monopole normalized to 1 at $t=M_{R}^{2}$, etc.). The coupling constants are either determined in the fit to the $N N$ scattering and bound state data or are taken from other sources, the form factor cutoffs always having to be determined from the fit. It is obvious from these considerations that such heavymeson exchanges generate four-nucleon terms with zero, two, four, etc., derivatives.

In Appendix B, we collect the explicit formulas for scalar, pseudoscalar, and vector meson exchanges, which can be applied to any of the OBE potentials by using the appropriate masses and coupling constants (and should be used instead of the symbolic formulas given before). As a typical example for an OBE potential we consider the Bonn-B variant [37]. Its short-range part is build from scalar $(\sigma, \delta)$, pseudoscalar $(\eta)$, and vector meson $(\rho, \omega)$ exchanges, and the pertinent contributions to the LECs $C_{i}, \widetilde{C}_{i}$ are listed in Table II. Another (more recent) OBE potential is the Nijmegen 93 potential (denoted Nijm-93) [19]. The Nijmegen 93 potential is particular since it also includes mesons with strange quarks but total strangeness zero [like the scalar $\epsilon(1300)$ or the $\phi(1020)$ mesons] and a low-energy representation of the Pomeron, which usually is needed to describe very high energetic proton-proton scattering. SU(3) flavor symmetry is imposed so that certain couplings are linked. The various contributions to the LECs are displayed in Table III. Some of the individual terms are unnaturally large (in particular those from the Pomeron), but the total contribution of the scalar sector is quite similar to the ones in the Bonn-B potential, as comparison of Tables II and III reveals.

The vector meson contributions $(\rho, \omega)$ are very similar for both potentials. The resulting LECs for these two OBE potentials are summarized in Table IV. Note that the variation in the values of the LECs resulting from taking different potentials is much smaller than the range over which the $C_{i}$ 's are distributed. Further, the somewhat large spread for the $C_{1_{1}}$ is possibly due to the fact that in traditional OBE models the phase shift ${ }^{1} P_{1}$ is usually not too well described. This

\begin{tabular}{|c|c|c|c|c|c|c|}
\hline LEC & $\eta$ & $\sigma$ & $\delta$ & $\omega$ & $\rho$ & Sum \\
\hline$\widetilde{C}_{{ } S_{0}}$ & 0.000 & -0.392 & -0.023 & 0.287 & 0.011 & -0.117 \\
\hline$C_{1_{S}}$ & 0.033 & 1.513 & 0.036 & -0.560 & 0.254 & 1.276 \\
\hline$\widetilde{C}_{{ }^{3} S_{1}}$ & 0.000 & -0.424 & 0.070 & 0.287 & -0.034 & -0.101 \\
\hline$C_{3} S_{1}$ & -0.011 & 1.030 & -0.108 & -0.777 & 0.526 & 0.660 \\
\hline$C_{\epsilon_{1}}$ & -0.032 & 0.000 & 0.000 & 0.077 & -0.455 & -0.410 \\
\hline$C_{{ }_{1} P_{1}}$ & -0.022 & -0.607 & 0.059 & 0.536 & 0.488 & 0.454 \\
\hline$C_{3 P_{0}}$ & -0.067 & -0.786 & -0.011 & 1.187 & 0.597 & 0.921 \\
\hline$C_{3 P_{1}}$ & 0.045 & -0.860 & -0.015 & 0.753 & 0.003 & -0.075 \\
\hline$C_{3} P_{2}$ & 0.000 & -1.008 & -0.024 & 0.536 & 0.101 & -0.396 \\
\hline
\end{tabular}
can be related to higher-order $\pi$ exchanges. Thus, a spread in

TABLE II. Contributions of the various boson exchanges to the LECs for the Bonn-B potential and the corresponding sum. The $\widetilde{C}_{i}$ are in $10^{4} \mathrm{GeV}^{-2}$ and the $C_{i}$ in $10^{4} \mathrm{GeV}^{-4}$. 
TABLE III. Contributions of the various boson exchanges to the LECs for the Nijmegen 93 potential. Pseudoscalars, $\eta, \eta^{\prime}$; vectors, $\rho, \omega, \phi$; scalars, $a_{0}, \epsilon, f_{0}, a_{2}$; Pomeron. The $\widetilde{C}_{i}$ are in $10^{4} \mathrm{GeV}^{-2}$ and the $C_{i}$ in $10^{4} \mathrm{GeV}^{-4}$.

\begin{tabular}{lcccccccccc}
\hline \hline LEC & $\eta$ & $\eta^{\prime}$ & $\rho$ & $\omega$ & $\phi$ & $a_{0}$ & $\epsilon$ & $f_{0}$ & $a_{2}$ & Pom. \\
\hline$\widetilde{C}_{{ } S_{0}}$ & 0.000 & 0.000 & 0.020 & 0.237 & 0.001 & -0.031 & -0.578 & -0.201 & 0.001 & 0.490 \\
$C{ }_{1} S_{0}$ & 0.041 & 0.013 & 0.191 & -0.445 & -0.002 & 0.134 & 3.461 & 0.867 & -0.005 & -2.829 \\
$\widetilde{C}_{{ }} S_{1}$ & 0.000 & 0.000 & -0.055 & 0.237 & 0.001 & 0.094 & -0.578 & -0.201 & -0.003 & 0.490 \\
$C_{{ }^{3}} S_{1}$ & -0.014 & -0.004 & 0.550 & -0.700 & -0.003 & -0.403 & 3.461 & 0.867 & 0.015 & -2.829 \\
$C_{\epsilon_{1}}$ & -0.038 & -0.012 & -0.383 & 0.090 & 0.000 & 0.000 & 0.000 & 0.000 & 0.000 & 0.000 \\
$C_{1} P_{1}$ & -0.027 & -0.009 & 0.431 & 0.423 & 0.002 & 0.250 & -2.194 & -0.539 & -0.010 & 1.791 \\
$C_{{ }_{3} P_{0}}$ & -0.082 & -0.026 & 0.645 & 1.167 & 0.006 & -0.072 & -1.985 & -0.466 & 0.003 & 1.613 \\
$C_{{ }_{3} P_{1}}$ & 0.054 & 0.017 & 0.032 & 0.660 & 0.003 & -0.078 & -2.087 & -0.501 & 0.003 & 1.700 \\
$C_{{ }_{3} P_{2}}$ & 0.000 & 0.000 & 0.114 & 0.457 & 0.002 & -0.090 & -2.308 & -0.579 & 0.003 & 1.887 \\
\hline \hline
\end{tabular}

$C_{1_{P_{1}}}$ is not surprising. The OBE models under consideration exhibit differences in the mixing parameter $\epsilon_{1}$, indicating variations in the tensor force. Thus a somewhat larger spread in $C_{\epsilon_{1}}$ and $C_{3_{S_{1}}}$ should be expected. Now comparing to the LECs determined using the chiral EFT potential listed in Table I we see a striking similarity. This result is somewhat surprising, because the phenomenological potential models are not constructed based on any power counting nor chiral symmetry, plus in many cases contain quantum field theoretically ill-defined form factors. Still, it is gratifying to see that the contact part of the $N N$ potential does not depend on how the short-distance physics is parametrized. To our knowledge, this is the first time that a direct link between the Weinberg program of systematically deriving nuclear forces from chiral Lagrangians to these phenomenologically successful potentials has been achieved in a truly quantitative manner.

There exists also a different class of potentials, which are constructed to give $\chi^{2} /$ datum $\simeq 1$ fits to the $N N$ data base like the high-precision charge-dependent CD-Bonn 2000 potential [18]. It contains two scalar-isoscalar mesons in each partial wave up to angular momentum $J=5$ with the mass and coupling constant of the second $\sigma$ fine-tuned in any partial wave. The other high-precision potentials are the
Nijmegen I,II [19] as well as the Argonne V18 (AV-18) [38] potentials. For the former, one-pion exchange is supplemented by heavy-boson exchanges with adjustable parameters that are fitted for all (low) partial waves separately. The AV-18 potential starts from a very general operator structure in coordinate space and has fit functions for all these various operators. Note that we have switched off the various electromagnetic corrections implemented in the AV-18 potential code. Such potentials can also be expanded in terms of fournucleon contact operators with increasing dimension. We do not give the details here but only mention that we have done this using numerical methods. The corresponding LECs $C_{i}$ and $\widetilde{C}_{i}$ are also listed in Table IV. Again the resulting LECs are very similar to each other, especially the larger values. The smaller ones show now a larger relative variation. One has to conclude that on this level of comparison (see introductory remarks) the LECs of the effective chiral forces cannot be determined quantitatively from the high-precision potentials but the qualitative similarity is remarkable and especially the linkage to the OBE potentials may carry a physical message. In Fig. 4 we give a graphical representation of the LECs obtained in EFT (cf. Table I) compared with the results from the six potential models considered here (see Table IV). Note that (a) the uncertainties for the LECs deter-

TABLE IV. Results for the LECs for various OBE and other types of potentials as explained in the text. The so-called high-precision potentials are marked by an asterisk. The $\widetilde{C}_{i}$ are in $10^{4} \mathrm{GeV}^{-2}$ and the $C_{i}$ in $10^{4} \mathrm{GeV}^{-4}$.

\begin{tabular}{|c|c|c|c|c|c|c|}
\hline LEC & Bonn-B & CD-Bonn* & Nijm-93 & Nijm-I* & Nijm-II* & AV-18* \\
\hline$\widetilde{C}_{{ }_{1} S_{0}}$ & -0.117 & -0.140 & -0.061 & -0.137 & -0.091 & -0.037 \\
\hline$C{ }^{1} S_{0}$ & 1.276 & 1.388 & 1.426 & 1.391 & 1.357 & 1.409 \\
\hline$\widetilde{C}_{3 S_{1}}$ & -0.101 & -0.103 & -0.014 & -0.058 & 0.029 & 0.026 \\
\hline$C_{3 S_{1}}$ & 0.660 & 0.869 & 0.940 & 0.762 & 0.795 & 0.867 \\
\hline$C_{\epsilon_{1}}$ & -0.410 & -0.315 & -0.343 & -0.221 & -0.241 & -0.226 \\
\hline$C_{{ }_{1} P_{1}}^{1}$ & 0.454 & 0.228 & 0.119 & 0.328 & 0.401 & 0.290 \\
\hline$C_{3} P_{0}$ & 0.921 & 0.956 & 0.802 & 0.802 & 0.949 & 0.723 \\
\hline$C_{3} P_{1}$ & -0.075 & -0.051 & -0.197 & -0.059 & -0.075 & 0.067 \\
\hline $\mathrm{C}_{3} \mathrm{P}_{2}$ & -0.396 & -0.451 & -0.513 & -0.453 & -0.451 & -0.467 \\
\hline
\end{tabular}




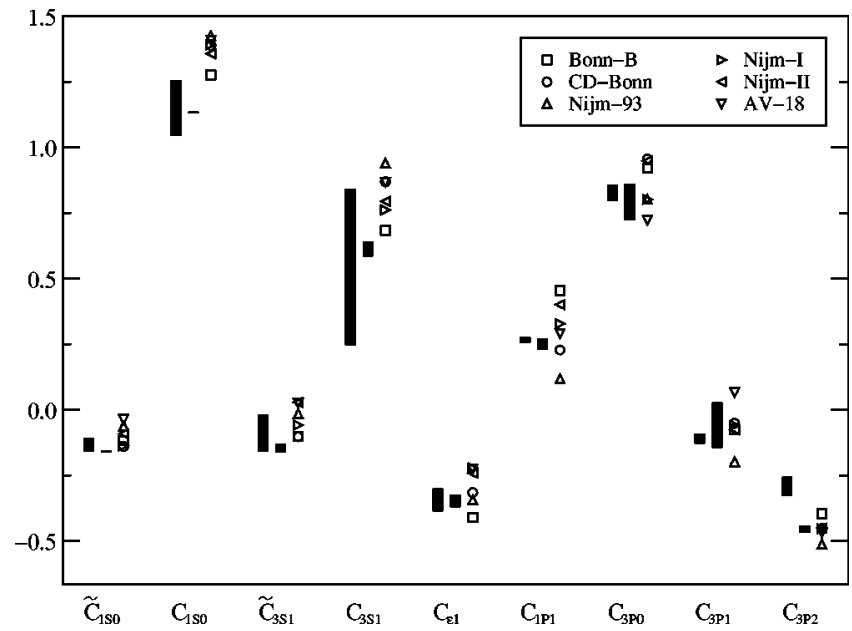

FIG. 4. LECs from phenomenological models and chiral EFT. The left-most band refers to NLO (the length reflects the variation with the cutoff), the middle bar is NNLO*, and the symbols correspond to the indicated potentials (see inset). The $\widetilde{C}_{i}$ are in $10^{4} \mathrm{GeV}^{-2}$ and the $C_{i}$ in $10^{4} \mathrm{GeV}^{-4}$.

mined from different $\mathrm{OBE}$ and high-precision potentials are small [typically, about 0.3 in the corresponding units (see Table I)] compared to the range of variation of the LECs (from -0.5 to 1.5 ) and (b) the uncertainties for the LECs determined in EFT are in most cases smaller than the band spanned by the potential models (even if one only includes the high-precision ones).

\section{NATURALNESS OF THE LECs AND WIGNER SYMMETRY}

First, we wish to investigate whether the LECs determined in Sec. II are of natural size. In the present context of Weinberg power counting, dimensional scaling arguments allow one to express any term of the effective Lagrangian with nucleon and pion fields as well as derivative and pion mass insertions (for a derivation and further discussion, see, e.g., Ref. [25]) as

$$
\mathcal{L}=c_{\text {lmn }}\left(\frac{N^{\dagger}(\cdots) N}{f_{\pi}^{2} \Lambda_{\chi}}\right)^{l}\left(\frac{\pi}{f_{\pi}}\right)^{m}\left(\frac{\partial^{\mu}, M_{\pi}}{\Lambda_{\chi}}\right)^{n} f_{\pi}^{2} \Lambda_{\chi}^{2},
$$

where the $c_{l m n}$ are dimensionless numbers and $l, m, n$ are non-negative integers. Here, $2 l$ counts the number of nucleon fields, $m$ the number of pions, and $n$ the number of derivatives or pion mass insertions. All nucleon isospin operators and so on are nonessential to this formula and indicated by the ellipsis. Note that this naive power counting cannot be applied to cases with spurious bound states, as witnessed by the so-called limit cycle behavior [39,40,32]. Here, we only consider potentials with no such spurious bound states; thus the relevant scale for the four-nucleon interactions without derivatives $(l=2, m=n=0)$ is the inverse of the pion decay constant, $f_{\pi}=92.4 \mathrm{MeV}$, squared and two derivative terms $(l=2, m=0, n=2)$ are suppressed by two inverse powers of the chiral scale $\Lambda_{\chi} \simeq 1 \mathrm{GeV}$. For the LECs from the Lagrangian (3) naturalness thus amounts to

$$
C_{i} \sim \frac{c_{200}}{f_{\pi}^{2}} \quad(i=S, T), \quad C_{j} \sim \frac{c_{202}}{f_{\pi}^{2} \Lambda_{\chi}^{2}} \quad(i=1, \ldots, 7),
$$

and the $c_{200}$ and $c_{202}$ should be numbers of order one [if there is not some suppression due to some symmetry (see below)]. Such arguments, of course, cannot say anything about the signs of the LECs. Also, it is important to realize the prefactors that accompany the various terms of the Lagrangian. For example, there is a relative factor of 4 in the momentum space representation between terms $\sim \vec{q}^{2}=\left(\vec{p}^{\prime}\right.$ $-\vec{p})^{2}$ and $\sim \vec{k}^{2}=\left(\vec{p}^{\prime}+\vec{p}\right)^{2} / 4$. Such factors need to be accounted for. Consequently, we give in Table V the corresponding coefficients $c_{200}$ and $c_{202}$ of the LECs as deduced from our NLO and NNLO fits using Eqs. (5). Inspection of the table reveals that the numbers fluctuate between 0.3 and 3.5 , i.e., the values found for these LECs are indeed natural, with the notable exception of $f_{\pi}^{2} C_{T}$, which is much smaller than one [except for the upper limit of NLO cutoffs, which is already close to the edge of having stable fits (see also the discusion in Ref. [14])]. As just mentioned, symmetry can lead to the suppression (or enhancement) of certain coupling constants. In fact, 65 years ago Wigner [41] proposed that

TABLE V. Naturalness coefficients of the LECs at NLO and NNLO for the cutoff values $\Lambda$ $=500, \ldots, 600 \mathrm{MeV}$. The $f_{\pi}^{2} C_{S, T}$ and the $f_{\pi}^{2} \Lambda_{\chi}^{2} C_{i}$ are dimensionless.

\begin{tabular}{lcc}
\hline \hline & NLO & NNLO \\
\hline$f_{\pi}^{2} C_{S}$ & $-1.053, \ldots,-0.303$ & $-1.079, \ldots,-0.953$ \\
$f_{\pi}^{2} C_{T}$ & $-0.002, \ldots, 0.147$ & $0.002, \ldots, 0.040$ \\
\hline$f_{\pi}^{2} \Lambda_{\chi}^{2} C_{1}$ & $1.707, \ldots, 3.162$ & $3.143, \ldots, 2.665$ \\
$4 f_{\pi}^{2} \Lambda_{\chi}^{2} C_{2}$ & $1.348, \ldots, 3.246$ & $2.029, \ldots, 2.251$ \\
$f_{\pi}^{2} \Lambda_{\chi}^{2} C_{3}$ & $-0.047, \ldots,-0.315$ & $0.403, \ldots, 0.281$ \\
$4 f_{\pi}^{2} \Lambda_{\chi}^{2} C_{4}$ & $-0.583, \ldots,-0.933$ & $-0.364, \ldots,-0.428$ \\
$2 f_{\pi}^{2} \Lambda_{\chi}^{2} C_{5}$ & $2.418, \ldots, 2.314$ & $2.846, \ldots, 3.410$ \\
$f_{\pi}^{2} \Lambda_{\chi}^{2} C_{6}$ & $-0.385, \ldots,-0.651$ & $-0.728, \ldots,-0.668$ \\
$4 f_{\pi}^{2} \Lambda_{\chi}^{2} C_{7}$ & $-1.790, \ldots,-2.120$ & $-1.929, \ldots,-1.681$ \\
\hline \hline
\end{tabular}


SU(4) spin-isospin transformations are an approximate symmetry of the strong interactions. Such a transformation has the form

$$
\delta N=i \varepsilon_{\mu \nu} \sigma^{\mu} \tau^{\nu} N, \quad N=\left(\begin{array}{l}
p \\
n
\end{array}\right), \quad \mu, \nu=0,1,2,3
$$

with $\sigma^{\mu}=(1, \vec{\sigma}), \tau^{\nu}=(1, \vec{\tau})$, and $\varepsilon_{\mu \nu}$ are infinitesimal group parameters. This symmetry emerges in the large number of color limits of QCD [42] and thus features prominently in the nuclear forces derived from Skyrme-type models. It was recently shown [43] that in the limit where the $S$-wave scattering lengths $a_{1 S 0}$ and $a_{3 S 1}$ go to infinity, the leading terms in the EFT for strong $N N$ interactions (with pions treated perturbatively) are invariant under Wigner's SU(4) spinisospin transformations. This can be seen most easily from the leading four-nucleon operators as used here [see Eq. (3)]. In this basis, the first term is clearly invariant under Wigner transformations [cf Eq. (14)], whereas the second term $\sim C_{T}$ obviously breaks the SU(4) symmetry. In the Weinberg approach employed here, the leading order potential consists of these two four-nucleon operators supplemented by the onepion exchange. Still, the Wigner symmetry is kept intact to good precision since the resulting fit values for $C_{T}$ are sizably smaller than the corresponding ones for $C_{S}$ (see Table $\mathrm{V})$. Stated differently, $C_{T}$ is unnaturally small because of the Wigner symmetry. This can be understood from the fact that at very low energies, where one is essentially sensitive to the ( $S$-wave) scattering lengths, the pion-exchange contribution can be expanded in powers of momenta, leading to terms with at least two derivatives (see Appendix B). One thus effectively recovers the situation eluded to in Ref. [43]. However, for larger momenta (say of the order of the pion mass), the nonperturbative treatment of the pions as proposed by Weinberg is mandatory.

\section{CONCLUSIONS AND SUMMARY}

In this paper, we have investigated the low-energy constants with zero and two derivatives that appear in the fournucleon contact interactions of the chiral effective Lagrangian for the nucleon-nucleon forces. Our main findings can be summarized as follows:

(1) We have determined the LECs for the NLO and NNLO potentials, including the dominant chargedependence effect from the pion mass difference in the onepion exchange. To avoid the unphysical bound states at NNLO, we have argued that one has to subtract the $\Delta$ contribution from the dimension-two pion-nucleon LECs. This is in agreement with two-boson-exchange models, where the two-pion-exchange contribution is cancelled largely by $\pi \rho$ graphs.

(2) We have shown how to deduce similar type of contact operators from boson-exchange models in the limit of large meson masses. This allows us to calculate the LECs in terms of meson-nucleon coupling constants, meson masses, and (unobservable) cutoff masses. In a similar manner, one can examine the so-called high-precision potential models. We have found that in all cases, the LECs determined from these models have a striking similarity to the values found in EFT. This can be considered as a kind of resonance saturation.

(3) We have shown that with the exception of one dimension-zero coupling (the LEC $C_{T}$ ), all LECs are of natural size. The smallness of $C_{T}$ is due to Wigner's spinisospin symmetry, as was already pointed out for the case of a theory with pions integrated out or treated perturbatively.

Clearly, these findings have further-reaching consequences. On one side, they might allow us to further constrain models of the nucleon-nucleon interaction applicable at energies where the EFT description cannot be used. On the other hand, in case of external sources (such as photons) or multinucleon operators (as they appear, e.g., in the description of the three-body forces), these considerations will allow us to at least estimate novel LECs that will appear. In the latter case of three- and more-nucleon systems, performing a direct fit for new adjustable parameters (if the leading nonvanishing three-nucleon force is included) to $3 \mathrm{~N}$ observables will be a very expensive task with respect to computer power. Therefore it might be very helpful to have a rough estimation for the values of various couplings appearing in the $3 N$ force.

\section{ACKNOWLEDGMENTS}

We would like to thank Hiroyuki Kamada and Andreas Nogga for many helpful discussions and for providing us with computer codes of the various potentials.

\section{APPENDIX A: REDUCTION OF THE TWO-PION-EXCHANGE CONTRIBUTIONS}

As stated before, we have to add the contribution of the TPE to the LECs so as to be able to compare with the bosonexchange potentials. The explicit expressions for the renormalized TPE potential at NLO can be found in Ref. [17]. Expanding those in powers of $\vec{q}$ and $\vec{k}$ allows for a mapping on the spectroscopic LECs (of course, the TPE contains many other contributions, which are, however, of no relevance for this discussion). We get

$$
\begin{gathered}
\widetilde{C}_{{ }^{1} S_{0}}^{\mathrm{NLO}}=-\frac{1}{3} \widetilde{C}^{\mathrm{NLO}}=\frac{\left(1+4 g_{A}^{2}-8 g_{A}^{4}\right) M_{\pi}^{2}}{24 \pi f_{\pi}^{4}}, \\
C_{{ }^{1} S_{0}}^{\mathrm{NLO}}=\frac{2+17 g_{A}^{2}-88 g_{A}^{4}}{144 \pi f_{\pi}^{4}}, \\
C^{\mathrm{NLO}}=-\frac{2+17 g_{A}^{2}-40 g_{A}^{4}}{48 \pi f_{\pi}^{4}}, \\
C_{{ } S_{1}}^{\mathrm{NLO}}=-\frac{g_{A}^{4}}{4 \sqrt{2} \pi f_{\pi}^{4}}, \\
C_{{ }^{\mathrm{NLO}} P_{1}}^{\mathrm{NLO}}=\frac{2+17 g_{A}^{2}-16 g_{A}^{4}}{72 \pi f_{\pi}^{4}},
\end{gathered}
$$




$$
\begin{aligned}
& C_{{ }^{3} P_{0}}^{\mathrm{NLO}}=-\frac{2+17 g_{A}^{2}+74 g_{A}^{4}}{216 \pi f_{\pi}^{4}}, \\
& C_{{ }^{3} P_{1}}^{\mathrm{NLO}}=-\frac{2+17 g_{A}^{2}-61 g_{A}^{4}}{216 \pi f_{\pi}^{4}}, \\
& C_{{ }^{3} P_{2}}^{\mathrm{NLO}}=-\frac{2+17 g_{A}^{2}-7 g_{A}^{4}}{216 \pi f_{\pi}^{4}} .
\end{aligned}
$$

Note that in the chiral limit, the two leading contact interactions do not get renormalized by TPE. Furthermore, these expressions only depend on the lowest-order pion-nucleon coupling $\sim g_{A}$ (or, by virtue of the Goldberger-Treiman relation, on $\left.g_{\pi N N}\right)$. Similarly, we can give the additional TPE NNLO contributions to the various LECs (for an explicit expression of the renormalized NNLO TPE potential, see e.g. Ref. [14]),

$$
\begin{aligned}
& \widetilde{C}^{\mathrm{NNLO}}=\frac{g_{A}^{2}\left(-16+192 m\left(-2 c_{1}+c_{3}\right)+25 g_{A}^{2}\right) M_{\pi}^{3}}{256 m f_{\pi}^{4}}, \\
& \widetilde{C}^{\mathrm{NNLO}}=\frac{3 g_{A}^{2}\left(16+64 m\left(-2 c_{1}+c_{3}\right)-21 g_{A}^{2}\right) M_{\pi}^{3}}{256 m f_{\pi}^{4}}, \\
& C_{{ }_{1} S_{0}}^{\mathrm{NNLO}}=\frac{g_{A}^{2}\left(-368-192 m\left(10 c_{1}-11 c_{3}+4 c_{4}\right)+869 g_{A}^{2}\right) M_{\pi}}{3072 m f_{\pi}^{4}}, \\
& C^{\mathrm{NNLO}} \\
& =-\frac{g_{A}^{2}\left(16\left(-7+4 m\left(10 c_{1}-11 c_{3}+4 c_{4}\right)\right)+81 g_{A}^{2}\right) M_{\pi}}{1024 m f_{\pi}^{4}}, \\
& C_{\epsilon_{1}}^{\mathrm{NNLO}}=\frac{g_{A}^{2}\left(8+32 m c_{4}-7 g_{A}^{2}\right) M_{\pi}}{64 \sqrt{2} m f_{\pi}^{4}}, \\
& C_{{ }_{P_{1}}}^{\mathrm{NNLO}}=\frac{g_{A}^{2}\left(-368+64 m\left(10 c_{1}-11 c_{3}-12 c_{4}\right)+305 g_{A}^{2}\right) M_{\pi}}{1536 m f_{\pi}^{4}} \\
& C_{{ }^{3} P_{0}}^{\mathrm{NNLO}}=\frac{g_{A}^{2}\left(176+192 m\left(10 c_{1}-11 c_{3}-8 c_{4}\right)+691 g_{A}^{2}\right) M_{\pi}}{4608 m f_{\pi}^{4}}, \\
& C_{{ }^{3} P_{1}}^{\mathrm{NNLO}}=\frac{g_{A}^{2}\left(464+192 m\left(10 c_{1}-11 c_{3}+2 c_{4}\right)-545 g_{A}^{2}\right) M_{\pi}}{4608 m f_{\pi}^{4}}, \\
& C_{{ }_{3} P_{2}}^{\mathrm{NNLO}} \\
& =-\frac{g_{A}^{2}\left(112+192 m\left(-10 c_{1}+11 c_{3}+2 c_{4}\right)+281 g_{A}^{2}\right) M_{\pi}}{4608 m f_{\pi}^{4}},
\end{aligned}
$$

with $m$ the nucleon mass. These expressions depend on the dimension-two LECs $c_{1,3,4}$ as discussed before. We note that all these contributions vanish in the chiral limit.

\section{APPENDIX B: REDUCTION OF ONE-BOSON EXCHANGES}

Here, we give the explicit expression for scalar, pseudoscalar, and vector meson exchange contributions to fournucleon operators with zero or two derivatives, as depicted in Fig. 3. Note that we will also include $1 / \mathrm{m}$ as well as $1 / \mathrm{m}^{2}$ corrections, which are, strictly speaking, of higher orders in the power-counting scheme we are working with and not (or partly) present in the NLO and NNLO potentials. There is, however, no contradiction since adding or subtracting those terms from the potential would lead to changes smaller than the level of accuracy of our approach. The contributions for a particular OBE potential can be obtained by using the appropriate masses, and coupling constants (and form factors) employed there. To obtain the most general expressions, we include any form factor as

$$
F_{M}\left(\vec{q}^{2}\right)=\alpha_{1}+\alpha_{2} \frac{\vec{q}^{2}}{\Lambda_{M}^{2}}+\mathcal{O}\left(\vec{q}^{4}\right),
$$

where the coefficient $\alpha_{1}=1$ if the form factor is normalized to 1 at $\vec{q}^{2}=0$ or $\alpha_{1} \neq 1$ if the form factor is normalized to 1 at $\vec{q}^{2}=-M_{M}^{2}$ (with $M_{M}$ the mass of the meson under consideration), or it might include the meson-nucleon coupling constant $g_{M}$. We will give the generic expression where the corresponding vertices are written as $g_{M} F_{M}\left(\vec{q}^{2}\right)$, with $F_{M}$ expanded as just discussed. Note for the exchange of isovector bosons such as $\pi$ or the $\rho$, the given expressions have to be multiplied by a factor $\boldsymbol{\tau}_{1} \cdot \boldsymbol{\tau}_{2}$, leading to a factor of -3 for the $T=0$ potential considered here.

\section{Scalar meson exchange}

The Lagrangian for coupling of an scalar-isoscalar meson with mass $M_{S}$ and coupling constant $g_{S}$ reads

$$
\mathcal{L}_{S}=g_{S} \bar{\psi} \psi \phi,
$$

where $\psi$ denotes the relativistic nucleon field and $\phi$ the scalar meson [for an isovector, one simply replaces $\phi$ by $\boldsymbol{\tau} \cdot \boldsymbol{\phi}$, with $\tau^{k}(k=1,2,3)$ the usual Pauli isospin matrices]. In the nonrelativistic expansion, the momentum space expression for the corresponding exchange potential with a form factor (if applicable) characterized by the cutoff $\Lambda_{S}$ reads up to terms of order $1 / m^{2}$

$$
\begin{aligned}
V_{S}(\vec{q}, \vec{k})= & -\frac{g_{S}^{2}}{\vec{q}^{2}+M_{S}^{2}}\left[1-\frac{\vec{k}^{2}}{2 m^{2}}+\frac{\vec{q}^{2}}{8 m^{2}}\right. \\
& \left.-\frac{i}{2 m^{2}} \vec{S} \cdot(\vec{q} \times \vec{k})\right] F_{S}^{2}\left(\vec{q}^{2}\right),
\end{aligned}
$$

where $\vec{S}=\left(\vec{\sigma}_{1}+\vec{\sigma}_{2}\right) / 2$ is the total spin of the two-nucleon system. The fully relativistic form of this exchange can be 
found, e.g., in Ref. [37]. This gives the following contributions to the spectroscopic LECs:

$$
\begin{aligned}
& \widetilde{C}^{S}=\widetilde{C}_{{ }^{1} S_{0}}^{S}=-\frac{4 \pi g_{S}^{2} \alpha_{1}^{2}}{M_{S}^{2}}, \\
& C_{{ }^{1} S_{0}}^{S}=C_{{ }^{3} S_{1}}^{S}=\frac{4 \pi g_{S}^{2} \alpha_{1}\left(-2 M_{S}^{2} \alpha_{2}+\alpha_{1} \Lambda_{S}^{2}\right)}{M_{S}^{4} \Lambda_{S}^{2}}, \\
& C_{\epsilon_{1}}^{S}=0, \\
& C_{{ }^{1} P_{1}}^{S}=\frac{2 \pi g_{S}^{2} \alpha_{1}\left[\left(-4+M_{S}^{2} / m^{2}\right) \alpha_{1}+8 M_{S}^{2} \alpha_{2} \Lambda_{S}^{2}\right]}{3 M_{S}^{4}}, \\
& C_{{ }_{3} P_{0}}^{S}=\frac{2 \pi g_{S}^{2} \alpha_{1}\left[8 m^{2} M_{S}^{2} \alpha_{2}-\left(4 m^{2}-3 M_{S}^{2}\right) \alpha_{1} \Lambda_{S}^{2}\right]}{3 m^{2} M_{S}^{4} \Lambda_{S}^{2}}, \\
& C_{{ }_{3} P_{1}}^{S}=\frac{4 \pi g_{S}^{2} \alpha_{1}\left[\left(-2+M_{S}^{2} / m^{2}\right) \alpha_{1}+4 M_{S}^{2} \alpha_{2} \Lambda_{S}^{2}\right]}{3 M_{S}^{4}}, \\
& C_{{ }^{3} P_{2}}^{S}=\frac{8 \pi g_{S}^{2} \alpha_{1}\left(2 M_{S}^{2} \alpha_{2}-\alpha_{1} \Lambda_{S}^{2}\right)}{3 M_{S}^{4} \Lambda_{S}^{2}} .
\end{aligned}
$$

\section{Pseudoscalar meson exchange}

The Lagrangian for the coupling of a scalar-pseudoscalar meson with mass $M_{P}$ and coupling constant $g_{P}$ reads

$$
\mathcal{L}_{P}=-g_{P} \bar{\psi} i \gamma^{5} \psi \pi
$$

where $\pi$ denotes the pseudoscalar meson (for an isovector, one simply replaces $\pi$ by $\boldsymbol{\tau} \cdot \boldsymbol{\pi}$ ). This is the so-called pseudoscalar coupling. Equivalently, one can also use a derivative-type (pseudovector) coupling

$$
\mathcal{L}_{P}^{\prime}=-\frac{f_{P}}{M_{P}} \bar{\psi} \gamma^{5} \gamma^{\mu} \psi \partial_{\mu} \pi
$$

At tree level, these couplings are equivalent provided $g_{P} / m=f_{P} / M_{P}$. Of course, chiral symmetry enforces the derivative coupling for the Goldstone bosons. In the nonrela- tivistic expansion, the momentum space expression for the corresponding exchange potential with a form factor (if applicable) characterized by the cutoff $\Lambda_{P}$ reads up to terms of order $1 / m^{2}$

$$
V_{P}(\vec{q})=-\frac{g_{P}^{2}}{4 m^{2}} \frac{\left(\vec{\sigma}_{1} \cdot \vec{q}\right)\left(\vec{\sigma}_{2} \cdot \vec{q}\right)}{\vec{q}^{2}+M_{P}^{2}} F_{P}^{2}\left(\vec{q}^{2}\right)
$$

Again, the fully relativistic form of this exchange can be found, e.g., in Ref. [37]. This gives the following contributions to the spectroscopic LECs:

$$
\begin{gathered}
\widetilde{C}^{P}=\widetilde{C}_{{ }^{1} S_{S_{1}}}^{P}=0, \\
C_{{ }^{1} S_{0}}^{P}=-3 C_{{ }^{3} S_{1}}^{P}=\Gamma_{P}, \\
-\frac{3}{2 \sqrt{2}} C_{\epsilon_{1}}^{P}=-\frac{3}{2} C_{{ }^{1} P_{1}}^{P}=-\frac{1}{2} C^{P}=\frac{3}{4} C_{{ } P_{0}}^{P}=\Gamma_{P}, \\
C_{{ }^{3} P_{1}}^{P}=0, \\
{ }^{3} P_{2}
\end{gathered}
$$

with

$$
\Gamma_{P}=\frac{\pi g_{P}^{2} \alpha_{1}^{2}}{m^{2} M_{P}^{2}}
$$

\section{Vector meson exchange}

The Lagrangian for coupling of a vector meson with mass $M_{V}$ and coupling constants $g_{V}$ (vector coupling) and $f_{V}$ (tensor coupling) reads

$$
\mathcal{L}_{V}=-g_{V} \bar{\psi} \gamma^{\mu} \psi \phi_{\mu}-\frac{f_{V}}{4 m} \bar{\psi} \sigma^{\mu \nu} \psi\left(\partial_{\mu} \phi_{\nu}-\partial_{\nu} \phi_{\mu}\right),
$$

where $\phi_{\mu}$ denotes the isoscalar-vector meson (for an isovector, one simply replaces $\phi_{\mu}$ by $\boldsymbol{\tau} \cdot \boldsymbol{\phi}_{\mu}$ ). In the nonrelativistic expansion, the momentum space expression for the corresponding exchange potential with a form factor (if applicable) characterized by the cutoff $\Lambda_{V}$ reads up to terms of order $1 / m^{2}$

$$
\begin{aligned}
V_{V}(\vec{q}, \vec{k})= & \frac{1}{\vec{q}^{2}+M_{V}^{2}}\left\{g_{V}^{2}\left[1+\frac{3 \vec{k}^{2}}{2 m^{2}}-\frac{\vec{q}^{2}}{8 m^{2}}+\frac{3 i}{2 m^{2}} \vec{S} \cdot(\vec{q} \times \vec{k})-\vec{\sigma}_{1} \cdot \vec{\sigma}_{2} \frac{\vec{q}^{2}}{4 m^{2}}+\frac{1}{4 m^{2}}\left(\vec{\sigma}_{1} \cdot \vec{q}\right)\left(\overrightarrow{\sigma_{2}} \cdot \vec{q}\right)\right]\right. \\
& \left.+\frac{g_{V} f_{V}}{2 m}\left[-\frac{\vec{q}^{2}}{m}+\frac{4 i}{m} \vec{S} \cdot(\vec{q} \times \vec{k})-\vec{\sigma}_{1} \cdot \vec{\sigma}_{2} \frac{\vec{q}^{2}}{m}+\frac{1}{m}\left(\vec{\sigma}_{1} \cdot \vec{q}\right)\left(\vec{\sigma}_{2} \cdot \vec{q}\right)\right]+\frac{f_{V}^{2}}{4 m^{2}}\left[-\vec{\sigma}_{1} \cdot \vec{\sigma}_{2} \vec{q}^{2}+\left(\vec{\sigma}_{1} \cdot \vec{q}\right)\left(\vec{\sigma}_{2} \cdot \vec{q}\right)\right]\right\} F_{V}^{2}\left(\vec{q}^{2}\right)
\end{aligned}
$$

Again, the fully relativistic form of this exchange can be found, e.g., in Ref. [37]. This gives the following contributions to the spectroscopic LECs: 


$$
\begin{aligned}
& \widetilde{C}_{{ }^{\prime} S_{0}}^{V}=\widetilde{C}_{{ }^{3} S_{1}}^{V}=\frac{4 \pi g_{V}^{2} \alpha_{1}^{2}}{M_{V}^{2}} \\
& C_{{ }_{1} S_{0}}^{V}=\frac{\pi \alpha_{1}\left\{8 m^{2} g_{V}^{2} M_{V}^{2} \alpha_{2}-\left[4 m^{2} g_{V}^{2}-\left(2 f_{V}^{2}+2 f_{V} g_{V}+3 g_{V}^{2}\right) M_{V}^{2}\right] \alpha_{1} \Lambda_{V}^{2}\right\}}{m^{2} M_{V}^{4} \Lambda_{V}^{2}}, \\
& C_{{ }^{3} S_{1}}^{V}=\frac{\pi \alpha_{1}\left\{24 m^{2} g_{V}^{2} M_{V}^{2} \alpha_{2}-\left[12 m^{2} g_{V}^{2}+\left(2 f_{V}^{2}+10 f_{V} g_{V}-g_{V}^{2}\right) M_{V}^{2}\right] \alpha_{1} \Lambda_{V\}}^{2}\right\}}{3 m^{2} M_{V}^{4} \Lambda_{V}^{2}}, \\
& C_{\epsilon_{1}}^{V}=\frac{2 \sqrt{2} \pi\left(f_{V}+g_{V}\right)^{2} \alpha_{1}^{2}}{3 m^{2} M_{V}^{2}}, \\
& C_{{ }_{P_{1}}}^{V}=\frac{4 \pi \alpha_{1}\left\{-4 m^{2} g_{V}^{2} M_{V}^{2} \alpha_{2}+\left[2 m^{2} g_{V}^{2}-f_{V}\left(f_{V}+g_{V}\right) M_{V}^{2}\right] \alpha_{1} \Lambda_{V\}}^{2}\right\}}{3 m^{2} M_{V}^{4} \Lambda_{V}^{2}}, \\
& C_{{ }^{V} P_{0}}^{V}=\frac{4 \pi \alpha_{1}\left\{-4 m^{2} g_{V}^{2} M_{V}^{2} \alpha_{2}+\left[2 m^{2} g_{V}^{2}+\left(2 f_{V}^{2}+9 f_{V} g_{V}+6 g_{V}^{2}\right) M_{V}^{2}\right] \alpha_{1} \Lambda_{V}^{2}\right\}}{3 m^{2} M_{V}^{4} \Lambda_{V}^{2}}, \\
& C_{{ }^{V} P_{1}}^{V}=\frac{2 \pi \alpha_{1}\left(-8 m^{2} g_{V}^{2} M_{V}^{2} \alpha_{2}+\left\{-M_{V}^{2} f_{V}^{2}+4 g_{V}\left[m^{2} g_{V}+\left(f_{V}+g_{V}\right) M_{V}^{2}\right]\right\} \alpha_{1} \Lambda_{V}^{2}\right)}{3 m^{2} M_{V}^{4} \Lambda_{V}^{2}}, \\
& C_{{ }_{P_{2}}^{V}}^{V}=\frac{2 \pi \alpha_{1}\left[f_{V}^{2} M_{V}^{2} \alpha_{1} / m^{2}+4 g_{V}^{2}\left(\alpha_{1}-2 M_{V}^{2} \alpha_{2} / \Lambda_{V}^{2}\right)\right]}{3 M_{V}^{4}} .
\end{aligned}
$$

[1] S. Weinberg, Phys. Lett. B 251, 288 (1990); 363, 3 (1991).

[2] J. Gasser and H. Leutwyler, Nucl. Phys. B250, 465 (1985).

[3] S. Myint and C. Rebbi, Nucl. Phys. B421, 241 (1994).

[4] J. Heitger, R. Sommer, and H. Wettig, Nucl. Phys. B588, 377 (2000).

[5] G. Ecker et al., Nucl. Phys. B321, 311 (1991).

[6] J.F. Donoghue, C. Ramirez, and G. Valencia, Phys. Rev. D 39, 1947 (1989).

[7] S. Bellucci, J. Gasser, and M.E. Sainio, Nucl. Phys. B423, 80 (1994).

[8] R. Baur and R. Urech, Nucl. Phys. B499, 319 (1997).

[9] B. Moussallam, Nucl. Phys. B504, 381 (1997).

[10] G. Ecker, J. Kambor, and D. Wyler, Nucl. Phys. B394, 101 (1993).

[11] V. Bernard, N. Kaiser, and Ulf-G. Meißner, Nucl. Phys. A615, 483 (1997).

[12] V. Bernard, N. Kaiser, and Ulf-G. Meißner, Z. Phys. C: Part. Fields 70, 483 (1996); Phys. Lett. B 378, 337 (1996).

[13] B. Borasoy and Ulf-G. Meißner, Ann. Phys. (N.Y.) 254, 192 (1997).

[14] E. Epelbaum, W. Glöckle, and Ulf-G. Meißner, Nucl. Phys. A671, 295 (2000).

[15] E. Epelbaum, H. Kamada, A. Nogga, H. Witała, W. Glöckle, and Ulf-G. Meißner, nucl-th/0201064.

[16] C. Ordóñez, L. Ray, and U. van Kolck, Phys. Rev. C 53, 2086 (1996)
[17] E. Epelbaum, W. Glöckle, and Ulf-G. Meißner, Nucl. Phys. A637, 107 (1998).

[18] R. Machleidt, Phys. Rev. C 63, 024001 (2001).

[19] V.G.J. Stoks, R.A.M. Klomp, C.P.F. Terheggen, and J.J. de Swart, Phys. Rev. C 49, 2950 (1994).

[20] E. Epelbaum, W. Glöckle, A. Küger, and U.-G. Meißner, Nucl. Phys. A645, 413 (1999).

[21] E.E. van Faassen and J.A. Tjon, Phys. Rev. C 28, 2354 (1983).

[22] R. Machleidt, K. Holinde, and Ch. Elster, Phys. Rep. 149, 1 (1987).

[23] U. van Kolck, G.A. Miller, and D.-O. Riska, Phys. Lett. B 388, 679 (1996)

[24] U. van Kolck, J.L. Friar and T. Goldman, Phys. Lett. B 371, 169 (1996)

[25] J.L. Friar, Few Body Syst. 22, 161 (1997).

[26] P.G. Lepage, Lectures given at 9th Jorge Andre Swieca Summer School: Particles and Fields, Sao Paulo, Brazil, 1997, hep-ph/9706029.

[27] E. Epelbaum, doctoral thesis, published in Berichte des Forschungszentrum Jülich, No. 3803, 2000.

[28] M.E. Luke and A.V. Manohar, Phys. Lett. B 286, 348 (1992).

[29] N. Kaiser, R. Brockmann, and W. Weise, Nucl. Phys. A625, 758 (1997).

[30] M. Walzl, Ulf-G. Meißner, and E. Epelbaum, nucl-th/0010019, Nucl. Phys. A (to be published).

[31] E. Epelbaum et al., Phys. Rev. Lett. 86, 4787 (2001). 
[32] E. Epelbaum and Ulf-G. Meißner (unpublished).

[33] C.H. Hyun, T.-S. Park, and D.-P. Min, Phys. Lett. B 473, 6 (2000)

[34] P. Büttiker and Ulf-G. Meißner, Nucl. Phys. A668, 97 (2000).

[35] G. Janssen, K. Holinde, and J. Speth, Phys. Rev. C 54, 2218 (1996); K. Holinde, Prog. Part. Nucl. Phys. 66, 311 (1996).

[36] E. Epelbaum et al., in Mesons and Light Nuclei: 8th Conference, edited by J. Adam et al., AIP Conf. Proc. No. 603 (AIP, Melville, NY, 2001).

[37] R. Machleidt, Adv. Nucl. Phys. 19, 189 (1989).
[38] R.B. Wiringa, V.G.J. Stoks, and R. Schiavilla, Phys. Rev. C 51, 38 (1995).

[39] P. Bedaque, H.W. Hammer, and U. van Kolck, Phys. Rev. Lett. 82, 463 (1999).

[40] S.R. Beane et al., quant-ph/0010073.

[41] E. Wigner, Phys. Rev. 51, 106 (1937); 51, 947 (1937); 56, 519 (1939).

[42] D.B. Kaplan and M.J. Savage, Phys. Lett. B 365, 244 (1996); D.B. Kaplan and A.V. Manohar, Phys. Rev. C 56, 76 (1997).

[43] T. Mehen, I.W. Stewart, and M.B. Wise, Phys. Rev. Lett. 83, 931 (1999). 